\title{
Effects of Happiness on Mental Health
}

\author{
Dr. Gopal Chandra Mahakud ${ }^{1}$, Ritika Yadav ${ }^{2}$
}

\section{ABSTRACT:}

The concept of mental health comprised to the health conditions of people without suffering any mental or psychological problem such as stress, depression, anxiety and other form of psychic disorders. In this regard it can be said that no one is free from and psychological, psychophysical and psycho-social disorders from which we can derive that no one mentally healthy. But the concept of mental health defined free from the disorders those are prolonged and panic in nature. As the concept of mental health is subjective in nature, it varies from person to person. Besides free from the disorders, a person should pose some of the other positive characteristics to deal with the society effectively. Marry (1958) stated that, a person can be considered mentally healthy with the following characteristics such as (a) Positive attitudes toward himself/herself; (b) Realization of own potentialities through action; (c); Unification of in personality; (d) Degree of independence of social influences; (e) observations of the world around; and (f) Positive adapts to everyday life. Briefly, it can be said that positive mental health of the person make able to an individual to stand on his own two feet without making undue demands or impositions of others. In this regard the role of happiness in day to day life can make the individual more skilled to fight with different mental disorders. The present article is intended to find out the effects of happiness in day to day life in a social situation to deal with different mental disorders to make the individual mentally healthy and prosperous in life.

Keywords: Happiness, Positive mental health, Stress, Depression, Anxiety, Panic disorder

The concept of positive psychology stands on the concept different mental process especially for human. It stated that most of the people first think positive or think more positive and less negative. The positive domains of human psychology were earlier dominated by the domains of negative concept and psychology initially started with the concept of different mental problems and disorders. During the initial time of clinical psychology, and its role in dealing different problem of human life started searching to find out the negative causes related to life those are also responsible for disruptive behavior or maladjusted behavior. In this regard, very recently the contribution of Martin Seligman and his colleagues started to search the effects of positive attributes of human behavior which can make the people more positive, healthy, peaceful and long-lived.

\footnotetext{
${ }^{1}$ Assistant Professor, Department of Applied Psychology, University of Delhi, South Campus, New Delhi

${ }^{2}$ M. A. Student, Department of Applied Psychology, University of Delhi, South Campus, New Delhi 
In this regard the present article intends to find out some of the mental health problems, mental disorders and how happiness can be helpful to deal effectively with different mental problems and disorders and to lead a healthy and prosperous life.

Mental Health: All though the concept of mental health in India is not so popular is, still, most of the people in India, especially from cities are conscious about the concept of mental health. In other words, it can be said that earlier people were more concerned about their physical health and also believed that physical health only have the relationship with mental health such as happiness, means if someone will be physically healthy, the person is happy. Some concept also come from the Socio Economic status of the people, means if some are wealthy, the person is happy or living happily. Although the history of mental health is too old in western countries, the concept is still new in India. According to World Health Organization (WHO) "A healthy mind can represent a healthy body". From this importance of mental health of WHO and some of the other health sector organization and now days numbers of initiatives have been taken by the Government if India, Non-Governmental Organization, and other social bodies to deal various hindrances related to mental problems and mental disorders. In this regard numbers of psychologist, psychiatrist, sociologist and other professional have been working to maintain the society healthier by keeping in mind especially the mental health issues, still very few people have done the work in relation to happiness and its relation to different mental disorders. Further mental health can be defined as the pleasant state of psychological health or a state of mind without any mental disorders. In the other hand it can be said that psychological wellbeing is the state of mind where people are satisfied in, psychological, psychosocial and psychophysiological day to day life. In this context, the world health organization definition of can be illustrated. According to World Health Organisation (WHO) wellbeing is mostly subjective in nature and it is the condition includes subjective wellbeing, perceived self-efficacy, autonomy, competence, intergenerational dependence and self-actualization of one's intellectual and emotional potential.

Happiness: Positive psychology cannot be fulfilled without the discussion of Happiness, the main concern about the mental health. The introduction of positive psychology is not very old concept. In the year of 2000, Martin Seligman became president of the APA. He is the first person founded positive psychology, which is more popular worldwide today. The common theme of positive psychology talks about the positive emotions such as happiness, pleasure, wellbeing, joy, delighted and so on against some of the traditional concept such as depression, anxiety, stress anger, etc especially clinical psychology clinical psychology stands on. Happiness is a holistic ideal and a fundamental object of human existence, this regard, the World Health Organization is increasingly emphasizing happiness as a component of health. Happiness is a pleasurable and satisfying experience or it is a state of well being characterized by emotions ranging from contentment to intense joy. Happiness may produce a pleasant mood, positive emotions, well being and positive attitudes towards self and other. 
Happiness and Social relationships: close relationships are central to people throughout their lives (Baumeister \& leary, 1995). Some of the researchers (e.g. Arygle; 2001; Campbell, Converse, \& Rodgers, 1976) affirmed that social relationships have long been considered one of the strongest and most important predictors of happiness. The most important question in this concern is to say that whether happiness is subjective or objective. In many researches, especially in relationship studies happiness have been discussed as the Subjective Well-Being (SWB) where it can be said that happiness is not a constant criteria for every individual (Diener, 1984; Dinener et. al., 1999). In other words it can be said that happiness is a different concept for person to person.

The works of (Watson, 1930; Wilson, 1967; and Diener 1984) explored the role of various types and dimensions of close relationships and its relation to happiness. Similarly Argyle, (2001); Diener \& Biswas Diener, (2008) stated that happiness can be predicted from the point of attachment security, social support, and overall relationship quality. These findings have been observed across samples, age groups, research methods, and cultures. Secondly, although close relationships are consistent correlates with happiness, research shows that the quality - a pattern replicated across age and cultural groups. In their views they also stated that personality type and happiness have close relationship therefore some people are happy with relationship where as other are contradictory.

Happiness and Freedom: The relationship of freedom and happiness is qyite positive in nature. Freedom may be defined as the action; people do according to their own wish. But it is important to say that, freedom leads to more happiness when it is followed by the social norms and regulations. In some of the cases it is seen that a wise choice leads to great happiness than to taking any decision following the physical and short term pleasure. Therefore it can be said that, a wise freedom can ensure long-term happiness and is thus far more valuable than a short-term comfort such as safety. In this regard Benjamin Franklin (1818), one of the great proponents of freedom, stated that "They, who can give up essential liberty to obtain a little temporary safety, deserve neither liberty nor safety". From the statement of Benjamin Franklin, it can be said that taking the original meaning of freedom and liberty can give more pleasure and happiness to the individual in society.

Positive Emotions and its Relation to Happiness: According to attachment theory of Bowlbt (1973), it can be stated that person who are more interactive and have the strong feelings of attachment with the person effectively in the society leads to happiness than to In this regard the there is a strong association between attachment patterns and the experience of positive emotions. It is believed that by imparting a sense of safety and security, interactions with available and supportive attachment figures alleviate distress and evoke positive emotions such as relief, love and gratitude. With repeated positive interactions, the sense of attachment security gradually becomes associated in a person's mind with memories of positive experiences and emotions. As a result, the evocation of mental representations of attachment security by either 
external or internal stimuli for example the presence of a supportive other, visualizing the face of an attachment figure automatically causes a person to feel more relaxed, relieved, loved, and happy (Mikulincer \& Shaver, 2007a)

Further using well-validated priming techniques, Mikulincer, Hirschberger, Nachmias, and Gillath (2001) provided experimental evidence for the hypothesized link between attachment security and positive emotion. Measures of attachment anxiety and avoidance have also been correlated with measures of positive affect (e.g., Gilbert er al ., 2008; Shiota, Keltner, \& John, 2006). For example several studies have used the Positive and Negative Affect Schedule (PANAS), finding that attachment anxiety and avoidance are associated with lower positive affect scores. Similar associations have been observed in studies examining positive emotions during daily social interactions tracked over one or more weeks. Positive emotion and its relation to different types of attachment have found in many studies. For example, Sonnby-Borgstrom and Jonsson (2003) exposed people to pictures of happy and angry faces, assessed the activity of the participants' smile and frown muscles, and found that attachment-anxious individuals had more active "frown" muscles when viewing either happy or angry faces.

Attachment patterns and the psychological consequences of positive emotions: The psychological consequences of positive emotions are moderated by attachment orientations and are more likely to be found among people who score high on attachment security i.e. low on anxiety and avoidance. Attachment patterns are systematically related to general psychological well-being, positive emotions in the context of close relationships, emotional reactions and the consequences of positive emotions. Moreover, the effects of attachment patterns are consistent across samples and research methods and can be generalized across cultures (Shaver et at., 2010).

Negative emotions vs positive emotions and its relation to Happiness: Negative emotions are more noticeable. Although for every negative emotion we may find a corresponding positive emotion, negative emotions are more differentiated than positive emotions. It is common to say that, there are many ways to describe negative emotions but very few ways are ther through which the positive emotions can be described. Even in many culture and societies, it is quite difficult to define positive emotion but it is very easty to define negative emotions. The the traditional psychological concept focused more in negative attributes and less to the positive attributes. But we can deny that positive emotions have more intensive effect on human life than to negative emotions. Still, there is little doubt then that love is both more noticeable and powerful in our everyday life. The risks of responding inappropriately to negative events are greater than the risks of responding inappropriately to positive events, since negative events can kill us while positive events will merely enhance our well-being. In this regard it can be said that in most of the cases it is observed that there is a negative relationship exist between negative emotions and the happiness. In other words it can be said that people having negative emotions are less happy than to the people having positive emotions. 
People having positive emotions are typically considered themselves to be happy. The majority of people see themselves as above-average as far as most of their qualities are concerned and they rate their happiness as more than one-third above the middle of the scale. This means that our baseline is above average in the positive realm. A major advantage of such a rating is that it has motivational value, which is important in coping with our surroundings and which produces a strong immune response to infections. While sad and pessimistic people can better perceive and understand their environment, happy and optimistic people can better cope with their environment. In this regard Nico Frijda has suggested what he calls "The law of Hedonic Asymmetry" which states that pleasure is always contingent on change and disappears with continuous satisfaction, whereas pain may persist under persisting adverse conditions. Frijda further explains that emotions exist in order to signal situations in the world that require a response. Since in his view positive circumstances do not need a specific response, the emotional signaling system can be switched off.

The more noticeable nature of negative emotions does not imply that their impact on our life is greater. This issue is connected to our general view of human beings, and there are conflicting views on this matter. Thus, Spionoza argues: "A desire that arises from joy is stronger, other things being equal, than one that arises from sadness. "Spinoza connects this contention to his assumption that the very essence of a person is a striving to persevere in his being. Similarly, while Adam Ferguson claims that "pain, by its intenseness, its duration, or frequency, is greatly predominant". He thinks that "love and compassion are the most powerful principles in the human breast. "Ferguson believes that positive emotions are more compatible with our basic positive disposition toward others. Descartes' view is different: "Sadness is in some way primary and more necessary than joy, and hatred more necessary than love."

While an empirical investigation might determine whether negative emotions are more noticeable, it is more difficult to verify empirically which type of emotion has a greater impact on our life. However, in this issue I tend to agree with Spinoza and Ferguson.

To sum up, negative emotions are more noticeable than positive ones since attending to negative events is more important for our survival than attending to positive events. This does not necessarily imply that negative emotions have a more important role in our lives. The emotions that are more frequent and obvious are not obvious are not always the more significant. In any case, love in all its forms, seems to be one of the most significant and powerful emotion in our lives.

Different Mental Problems and its Relation to Happiness: The most common word of Mental Health confined with free of stress, Depression, Anxiety and other form of Neurotic and psychotic disorders. Numbers of studies already proved the association between negative emotion, negative affective behavior and its relationship with different mental disorders. In other words it can be said that negative emotions and negative affective behavioral components are the main cause of different mental health problems/disorders. Very few studies and literature established till date to find out the relationship of Happiness with different mental health issues 
and treatment. In other words few researches have proved the effects of happiness as an intervention for the treatment of mental disorders.

Happiness and Its Relations with Depression: In $21^{\text {st }}$ century, due to industrialization and intensive increasing changes of human life leads to the most common form of mental disorders that can be depression. According to World Health Organization (WHO) "Depression is a common mental disorder, characterized by sadness, loss of interest or pleasure, feelings of guilt or low self-worth, disturbed sleep or appetite, feelings of tiredness and poor concentration". The effects of depression may be long lasting or recurrent or it may be for a short period of time depending on the person's ability of management in the work place and in the family system. The serious effects of depression can lead the person towards death or suicide, if it is not cope effectively at the primary stage. Therefore it can be suggested that, when depression is in mild opr moderate stage psychological treatment can help to be free from depression but if level of depression is severe, it is better to suggest the client for medication along with psychotherapy. In most of the cases and from various studies it is observed that depression often starts at a young age. It affects women more often than men, and unemployed people are also at high risk. People with depression are not enjoying happiness. In this regard the study of Seligman's (2002) can be illustrated. The study was conducted on 577 people where happiness exercises were provided to the participants. In the present study it is found that happiness exercise decreases the level of depression among participants. Similarly the study of Phillips (1967) also stated that people suffering mental disorders are definitely unhappy in nature. Further the researcher stated that persons reporting mental disturbances as restlessness, inability to sleep, sour stomachs, and the like, would also report feelings of unhappiness. In this regard Phillips (1967) also suggested that social participation increase the happiness and happiness reduces the depression level of people suffering mental disorders.

Happiness and Its Relations with Anxiety: The common definition of anxiety is feelings of tension, worried thoughts. Due to anxiety there, some of the observable psycho-physiological symptoms can be observed such as increased blood pressure, heart palptation, sweating, nausea, irritation etc. in this regard it can be said that anxiety is a psycho-physiological state of mind which may be for a span of time or it may continue for a long period of time. Some of the other symptoms of anxiety disorders can be defined have recurring intrusive thoughts or concerns; avoid certain situations out of worry, prefer isolated situation, trembling, dizziness or a rapid heartbeat (DSM-IVTR). Therefore, it is found that, people suffering any type of anxiety disorders are not happy in their day to day life. Ekman, Davidson, Ricard, and Wallace, (2005) stated that more happy people are less prone to any anxiety disorders. Kendall, (1994) conducted a study on 47 participants aged 9 to 13 years where he employed happiness as one of treatment procedure for the treatment of psychosocial treatment for the students suffering anxiety disorders and found a positive result where happiness reduces the anxiety level of the participants. In a study by Clark, and Watson, (1991) also found the effect of happiness as a treatment for anxiety and depression disorders. Further in another study intervention study used 
happiness as an intervention technique conducted by O'connor, Dinan, and Cryan, (2011) also revealed that there is a positive effecst of happiness in treatment of anxiety disorders.

Stress and its Relation to Happiness: the concept of happiness for the people suffering stress is quite unnatural. Stress due to work place, family hassles, increasing day to day demands are some of the common reason which interfering the human happiness. In this regard KiecoltGlaser, Preacher, MacCallum, Malarkey, and Glaser, (2003) stated that those who are physically overwhelmed, mentally overwhelmed, or both by the needs of others do experience a stressful or burden that can have significant negative health consequences such as cardiac disorders, respiratory disorders and gastrointestinal disorders. It is not only affects to the person whoc is suffering stress, it also affect the mental health of the care givers and they also suffer similar results of stress what the person suffering. Numbers of studies already proved that, happiness can improve both physical and psychological disorders that due to stress. Current consensus indicates that helping behavior contributes to diminished depression rates in adolescents (Commission on Children at Risk, 2003). In this regard Post, (2005) affirmed that that a strong correlation exists between the well-being, happiness, health, and longevity of people who are emotionally kind and compassionate in their charitable helping activities - as long as they are not overwhelmed, and here world view may come into play.

Another study by Ryan and Deci (2000) stated that happiness contribute well-being and directly reduce the mental disorders such as stress depression and anxiety. Further the study of Dohrenwend, (2000) also indicates the effects of happiness on psychological stress disorders. The study was conducted with post traumatic stress disorders patients and found the result effective. The study of Sheldon, and Niemiec, (2006) also indicates that happiness develop self concept and self efficacy among the people and indirectly reduce the chances of psychological disorders. In a recent study by Aubert (2008, it is found that happiness reduces psychological stress and other emotional provoking disorders. In a study on cardiovascular patients Schwartz, Weinberger, and Singer (1981) also found that sadness and angry is the contradictory variables lead psychological stress and ultimately the heart and heart related disorders. In other hand happiness reduces the chances of cardiovascular disorders.

Conclusion: Happiness is one of the most important aspects of positive psychology. Although happiness considered as one of the important attribute of human emotions, its effect on mental disorders is found more favorable. The brief discussion of happiness and its effect on different mental disorders stated that although happiness is a subjective emotional component but still overall all happiness has a positive association with different mental disorders such as stress, anxiety and depression. In other words it can be said that people those are happy have less chance to prone to the mental disorders. They suffer less stress, depression and anxiety. As a result, they enjoy both psychological and physiological wellbeing. Happy people are also more sociable and enjoy quite good social wellbeing. In this regard these happy people are healthier 
than to the unhappy people. Therefore different techniques have been used by psychologist and social scientist to make people healthier and ultimately healthy physically, socially and mentally.

\section{REFERENCES}

Argyle M. (1987). The psychology of happiness. London:Methuen.

Argyle, M. (2001). The psychology of Happiness (2nd ed.). New York., NY: Routledge

Aubert, A. (2008). Psychosocial stress, emotions and cytokine-related disorders. Recent patents on inflammation \& allergy drug discovery, 2(2), 139-148.

Baumeister, R.F., \& Leary, M.R. (1995). The need to belong: Desire for interpersonal attachments as a fundamental human motivation. psychological Bulletin

Bay, C. (1965). The structure of freedom Stanford University Press, Stanford CL, USA.

Franklin, B. (1818). Memoirs of the Life and Writings of Benjamin Franklin (London: Henry Colburn, 1818) at 270.

Blokland, H.T (1997).Freedom and culture in western society Routledge, London,

Campbell, R.(1973). “ The Pursuit of Happiness.” Personalist

Clark, L. A., \& Watson, D. (1991). Tripartite model of anxiety and depression: psychometric evidence and taxonomic implications. Journal of abnormal psychology, 100(3), 316.

Commission on Children at Risk. (2003).Hardwired to connect: The new scientific case for authoritative communities.New York: Institute for American Values. Danner, D. D., Snowdon, D. A., \&

Demir , M. (2010). close relationships and happiness among emerging adults.

Dohrenwend, B. P. (2000). The role of adversity and stress in psychopathology: some evidence and its implications for theory and research. Journal of health and social behavior, 1-19.

Ekman, P., Davidson, R. J., Ricard, M., \& Wallace, B. A. (2005). Buddhist and psychological perspectives on emotions and well-being. Current Directions in Psychological Science, 14(2), 59-63.

Goldberg, C. (2006). Harvard's crowded course to happiness. Boston Globe http://www.boston.com/news/local/articles/2006/03/10/harvards_crowded_course_to_ha ppiness/

Kendall, P. C. (1994). Treating anxiety disorders in children: results of a randomized clinical trial. Journal of consulting and clinical psychology, 62(1), 100.

Kiecolt-Glaser, J. K., Preacher, K. J., MacCallum, R. C., Malarkey, W. B., \& Glaser, R. (2003). Chronic stress and age-related increases in the proinflammatory cytokine interleukin-6. Proceedings of the National Academy of Sciences, 100, 9090-9095.

Norman M. Bradburn and David Caplovitz, reports on happiness

O’connor, R. M., Dinan, T. G., \& Cryan, J. F. (2011). Little things on which happiness depends: microRNAs as novel therapeutic targets for the treatment of anxiety and depression. Molecular psychiatry, 17(4), 359-376.

Peterson, C. (2006). A Primer in Positive Psychology. New York: Oxford University Press.

Phillips, D. L. (1967). Mental health status, social participation, and happiness. Journal of Health and Social Behavior, 285-291. 
Post, S. G. (2005). Altruism, happiness, and health: It's good to be good. International journal of behavioral medicine, 12(2), 66-77.

Reis., H. T. (2001) Relationship experiences and emotional well-being. in C. D. Ryff \&B. H. Singer (Eds.) Emotion, Social relationships, and health.

Ryan, R. M., \& Deci, E. L. (2000). Self-determination theory and the facilitation of intrinsic motivation, social development, and well-being. American Psychologist, 55, 68-78.

Schwartz, G. E., Weinberger, D. A., \& Singer, J. A. (1981). Cardiovascular Differentiation of Happiness, Sadness, Anger, and Fear Following Imagery and Exercise1. Psychosomatic Medicine, 43(4), 343-364.

Seligman, M. E. P. (2002). Authentic happiness. New York: Free Press.

Sheldon, K. M., \& Niemiec, C. P. (2006). It's not just the amount that counts: balanced need satisfaction also affects well-being. Journal of personality and social psychology, 91(2), 331.

Snyder, C. R. \& Lopez, S. J. (Eds.) (2005). Handbook of Positive Psychology. New York: Oxford University Press.

UKHeadey, B. \& Wearing, A. (1992). Understanding happiness: a theory of subjective wellbeing Longman Cheshire, Melbourne, Australia: Sources-www.nakedcapitalism.com/.../keyto-happiness-is-freedom.

\section{Web Access:}

http://www.googleadservices.com.

https://mtgap.wordpress.com/2010/06/15/freedom-or-happiness

https://www.psychologytoday.com/basics/happiness 